\title{
Analyze Spontaneous Gestures for Emotional Stress State Recognition: A Micro-gesture Dataset and Analysis with Deep Learning
}

\author{
Haoyu Chen, Xin Liu, Xiaobai Li, Henglin Shi and Guoying Zhao* \\ Center for Machine Vision and Signal Analysis \\ University of Oulu, Finland
}

\begin{abstract}
Emotions are central for human intelligence and should have a similar role in AI. When it comes to emotion recognition, however, analysis cues for robots were mostly limited to human facial expressions and speech. As an alternative important non-verbal communicative fashion, the body gesture is proved to be capable of conveying emotional information which should gain more attention. Inspired by recent researches on micro-expressions, in this paper, we try to explore a specific group of gestures which are spontaneously and unconsciously elicited by inner feelings. These gestures are different from common gestures for facilitating communications or to express feelings on ones own initiative and always ignored in our daily life. This kind of subtle body movements is known as 'micro-gestures' (MGs). Work of interpreting the human hidden emotions via these specific gestural behaviors in unconstrained situations, however, is limited. It is because of an unclear correspondence between body movements and emotional states which need multidisciplinary efforts from computer science, psychology, and statistic researchers. To fill the gap, we built a novel Spontaneous Micro-Gesture (SMG) dataset containing 3,692 manually labeled gesture clips. The data collection from 40 participants was conducted through a story-telling game with two emotional state settings. In this paper, we explored the emotional gestures with a sign-based measurement. To verify the latent relationship between emotional states and MGs, we proposed a framework that encodes the objective gestures to a Bayesian network to infer the subjective emotional states. Our experimental results revealed that, most of the participants would do 'micro-gestures' spontaneously to relieve their mental strains. We also carried out a human test on ordinary and trained people for comparison. The performance of both our framework and human beings was evaluated on 142 testing instances (71 for each emotional state) by subject-independent testing. To authors' best knowledge, this is the first presented MG dataset. Results showed that the proposed MG recognition method achieved promising performance. We also showed that MGs could be helpful cues for the recognition of hidden emotional states.
\end{abstract}

\section{INTRODUCTION}

Emotions are central for human intelligence and should have a similar role in AI. When it comes to the emotion analysis and the emotion recognition, most previous works focused more on facial expression, speech and physiological signal (e.g., heartbeats) analysis, while body gestures were usually just used as a minor or complementary clue for

\footnotetext{
* Corresponding author

H. Chen, X. Liu, X. Li, H. Shi and G. Zhao are with the Center for Machine Vision and Signal Analysis, University of Oulu, Oulu 90014, Finland. E-mail:chen.haoyu, xin.liu, xiaobai.li, henglin.shi, guoying.zhao@oulu.fi
}

emotion recognition [21], [31], [14], [15], [13], as shown in Table I.

Over the past decade, gestures have been intensively studied and analyzed for other purposes, e.g., activity or sports recognition, sign-language recognition. Those gestures are commonly used for purposes to regulate interaction, to communicate a specific meaning, to greet, etc. According to [27], they are also known as illustrator gestures or iconic gestures. However, only limited works [16] devoted to using gestural behaviors to interpret human emotion or affection.

As a matter of fact, as an important non-verbal communicative cue, gestures are proved to be capable of conveying human's emotional information. On one side, some gestures can help people to explicitly express emotions or feelings in our daily life. On the other side, a special group of gestures can be used as helpful cues to reveal concealed or suppressed emotions. As this kind of gestures is typically performed unconsciously and unintentionally, they can reveal humanbeings' hidden emotional status which is the emotional status that people express intentionally. In [26] and [19], these body gestures that implies people's hidden emotion status (rather than ordinary emotions) were referred as the Micro-Gesture (MG). Moreover, psychological studies [23] [12] [1] already showed that MGs can be more reliable emotion indicators which may perform even better than facial expressions or acoustic clues.

The name of the micro-gesture (MG) comes from its related concept of micro-expressions [20] [30] which are subtle facial muscular movements, while MG indicates subtle movements of body parts other than the face to reveal hidden emotions. According to [17], MG covers a wide group of gestures including self-manipulation movements (e.g., scratching head and hand rubbing), manipulation of objects (e.g., playing with clothes), and self-protection gestures (e.g., folding arms or moving legs). MG samples are shown in the left part of Figure 2. All MGs share one important attribute, which differentiates them from nonmicro-gestures: they are not performed for any illustrative or communicational purposes at all, they are spontaneous or involuntary movements that the body responses to the onset of certain stimuli, especially negative ones. Meanwhile, ordinary gestures are usually performed on purpose of facilitating communications, e.g., to illustrate specific semantic meanings or to explicitly express one's feelings or attitudes, which are referred as illustrator gestures or iconic gestures (one sample is shown in Figure 2). This means people 


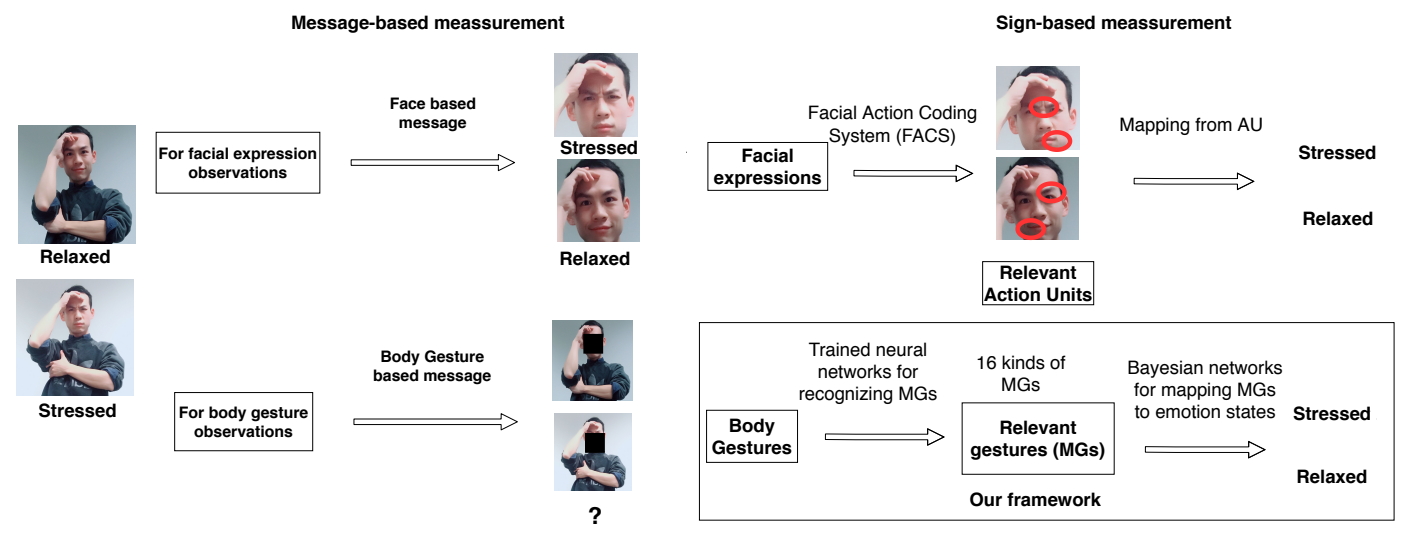

Fig. 1. A comparison of message-based measurement and sign-based measurement for emotion state recognition with different observations (facial expression and body gestures). In the previous work, the message-based measurement is commonly used, which means gestures are directly mapped to emotions. However, as we can see in the figure, different emotional states may share the same gesture.

\begin{tabular}{lclclcc}
\hline Dataset name & Subjects & Instances & Emotions & Gestures & Resources & Gestures performed \\
\hline SMG 2018 (our work) & $\mathbf{4 0}$ & $\mathbf{3 6 9 2}$ & $\mathbf{2}$ & $\mathbf{1 7}$ & RGBD+skeleton & Spontaneous \\
ICD-Navarasas-2018 [21] & 12 & 35 & 8 & 8 & RGBD & Semi-Enacted \\
FG-RGBD-2014 [31] & 12 & 1920 & 10 & 10 & RGBD & Enacted \\
BFU-2013 [14] & 29 & 60 & 2 & 2 & Grey Images & Enacted \\
FABO-2006 [15] & 23 & 206 & 9 & 72 & RGB & Semi-Enacted \\
Genova-2006 [13] & 10 & 240 & 8 & 8 & RGB & Enacted \\
\hline
\end{tabular}

A REVIEW OF RELEVANT DATABASES FOR EMOTION RECOGNITION. NOTE THAT IN ALL THE PREVIOUS DATABASES, GESTURES ONLY SERVE AS AN ADDITION TO FACIAL EXPRESSIONS. THERE IS NO PARTICULAR STUDY CONDUCTED TO SOLELY USE MICRO-GESTURES FOR EMOTION RECOGNITION, APART FROM THE CURRENT WORK WITH THE SMG DATASET.

perform ordinary (non-micro) gestures for the purpose of being seen and understood by others, in order to better express themselves. For example, during a job interview the employer asks a question that the interviewee cannot answer, he or she might keep smiling but spontaneously starts to rub hands because of nervousness. The interviewee certainly does not rub hands on purpose to let the employer see that he or she is getting nervous, it is just a subconscious response of the body to ease the stress. This is one typical scenario when MGs occur. Psychological studies [24] showed that MGs are important clues to reveal people's hidden emotional status, especially for negative feelings such as stress, nervousness, and fear. Trained specialists [22] can use these body clues to detect suspicious behaviors, e.g., someone is feeling stress and getting nervous. But for ordinary people, since MGs are mostly unobtrusive and subtle in magnitude, they are usually neglected in our daily interactions.

In this work, we want to explore whether computers can be trained to perform as experts, i.e., to catch MGs for understanding people's hidden emotional status. To be more specific, we propose methods to detect the occurrence of MGs when people are conducting tasks at different stress states. Then the recognized MGs are used as clues to recognize people's hidden emotional states (stress states). Automated emotion recognition with MGs has great potential in applications including social media, human-computer interaction, public safety, and health care [18].

Several challenging issues impede the progress of emotion recognition using MGs. First of all, a gold standard for emotion measurement through bodily gestures is absent. In facial expressions-based emotion recognition, two standard measurements are used. One is the message-based measurement that maps each facial expression to one certain emotion. However, there is no such direct mapping between gestures and emotional states. For example, people may perform the same movement in different emotional states as shown in Figure 1. The other one is sign-based measurement [5] such as Facial Action Coding System (FACS) [11] that encodes objective signs into corresponding emotions. Signbased measurement is suitable for gesture-based emotion recognition, but few efforts have been made so far [25]. Secondly, the degree of freedom of an articulated pose is considerably high, and the level of heterogeneity is also relatively staggering even in the same gesture class. Lastly, working with MGs in unconstrained data brings additional technical obstacles. For example, MGs are relatively subtle body movements that can easily be submerged in other unrelated body movements; accurate recognition and localization of MGs are critical for later emotion recognition.

We try to tackle the above problems in this paper and investigate the computability of hidden emotional states via MGs from the collected data. Here we summarize main contributions as follows.

1) To the best of authors' knowledge, this is the first attempt to investigate MGs with machine vision technologies for hidden emotion analysis. 
2) We built a new dataset, which includes spontaneous emotional gestures elicited from participants under two different emotional states.

3) For recognizing emotional states with gestures, we proposed a novel sign-based framework which uses deep-learning frameworks to detect MGs and then infers participates' hidden emotional stress states with Bayesian networks.

We got promising results using MGs for hidden emotional states recognition which exceeds common human observers by $12.6 \%$. This study is timely for filling the gap of the datasets specifically for studying the relationship of gestures and hidden emotional states. Conventions could be revealed and established for MG recognition, and for hidden emotional status recognition with MGs.

\section{The Spontaneous Micro-Gesture Dataset (SMG)}

Emotion-based human action and body gesture databases have been proposed in the past decade as mentioned in the Introduction section. However, emotional gestures in most of the previous databases were all designed deliberately, or say, the participants were asked to perform some certain and contrived emotions instead of spontaneously performing behaviours occurred in natural communication situations. Besides, few of those gestures were collected as the involuntary behaviors that embody hidden emotions which can better reveal the true human inner status. We built a new SMG dataset to fill the blank. The main characteristics of this dataset are: 1) Gestures should be spontaneous according to people's genuine feelings. 2) Participants should be exposed to different intensive tasks which correspond to different emotional states while they try to inhibit their emotions. 3) The induced emotional states should be different, while the tasks should not be too complicated for participants to avoid stagy behaviours. To this end, we design the experimental process for building the SMG dataset as follows.

\section{A. Two emotional stress states}

Firstly, to focus on the relationship between gestures and emotions instead of emotions themselves, we decide to choose only two emotional states. We choose two conversational tasks to simulate two distinct emotional states: the relaxed emotional state (RES) and the stressed emotional state (SES). More precisely, for the RES task, we provided participants with a short story, and they simply needed to repeat the contents of the story. For the SES task, participants only got a story title and they needed to make up a story offthe-cut according to this title and pretended that he or she knew the content. Compared with RES of "repeating a story", the other task of "making up a fake story" is certainly more difficult and involve higher cognitive load, which should induce higher stress level as SES.

\section{B. Data collection}

During data collection, one examiner was introduced, and participants needed to confirm the examiner that they were telling a real story (no matter what states they are in). This setting is vital for the collection of MGs because it means participants have to hide their real emotions, especially for those who are in SES. None of the participants was informed beforehand about the actual purpose of our research in case that participants may inhibit or deliberately adjust their body gestures and behaviors.

The data collection was conducted in a private laboratory. The illumination condition was indoor with normal daylight lamps. During the recording, participants were asked to stand in front of a Kinect V2 sensor with a recording speed of 28 frames per second. The collection was held with two participants at every turn. Two different tasks were assigned to the two participants separately, and one examiner observed them behind the scene. No restriction was given about the performance, and they were free to behave as they do everyday. Four modalities were recorded of the participants' behaviors: RGB, silhouette, depth videos, and skeleton coordinates as shown in Figure 2 (b). Skeleton coordinate information consists of tracks of 25 skeleton joints.

\section{Labeling hidden emotions and MGs}

The labeling of hidden emotion states was straightforward, all the story-repeating processes of the participants were labeled as the RES, and the processes of making-up fake stories were labeled as the SES. When it comes to the MG labeling, procedures were more tortuous. Before labeling the MGs from recording sequences, we referenced relevant literature of sentiment-orientated gestures [22], [3], [2], [8], [6], [9], [10], [7] and summarized 16 commonly accepted gestures that could be regarded as MGs. They are listed in Figure 2 (a). 'Non-micro-gestures' were also labeled as an independent category. The statistics of all gestures is shown in Table II.

\section{Dataset composition}

The final SMG dataset comprises of 40 sequences (821,056 frames in total, more than 8 hours) from 40 participants (each sequence lasts for around 15 minutes) with RGB, depth, silhouette video streams, and skeleton data. 40 participants were recruited from a European university with multicultural backgrounds (16 countries), 27 males and 13 females with an average age of 25 . It contains 71 RES instances and 71 SES instances. Lengths of those instances range from two to five minutes. Each participant contributes two instances or four instances (half are RESs and half are SESs). 3,692 gesture clips were labeled manually by five persons via a voting strategy. The average length of those MGs is 51.3 frames. The distribution of MGs in the two emotional states is shown in Table II. It can be seen that samples are severely unbalanced. There are 1,146 samples of 'Moving legs' while only 11 of 'Pulling shirt collars'.

\section{DATA ANALYSIS}

In this section, we report a relevant statistical analysis of the proposed dataset. A novel sign-based framework is 


\begin{tabular}{|c|c|c|c|c|c|c|c|}
\hline ID & Description & $\begin{array}{l}\text { Total } \\
\text { number }\end{array}$ & $\begin{array}{l}\text { Number in } \\
\text { SES/RES }\end{array}$ & ID & Description & $\begin{array}{l}\text { Total } \\
\text { number }\end{array}$ & $\begin{array}{l}\text { Number in } \\
\text { SES/RES }\end{array}$ \\
\hline 1 & Turtling neck and shoulder & 56 & $37 / 19$ & 10 & Crossing legs & 50 & $27 / 23$ \\
\hline 2 & Rubbing eyes and forehead & 51 & $33 / 18$ & 11 & $\begin{array}{l}\text { scratching some part of body } \\
\text { (other than face,head and fingers) }\end{array}$ & 24 & $17 / 7$ \\
\hline 3 & Folding arms & 197 & $134 / 63$ & 12 & $\begin{array}{l}\text { Scratching or touching facial parts } \\
\text { (chin, noses, ears)other than eyes }\end{array}$ & 220 & $134 / 86$ \\
\hline 4 & Touching or covering suprasternal notch & 11 & $6 / 5$ & 13 & Playing or adjusting hair & 64 & $42 / 22$ \\
\hline 5 & Moving legs & 1146 & $709 / 437$ & 14 & Hold arms & 69 & $44 / 25$ \\
\hline 6 & Touching or scratching neck & 24 & $12 / 12$ & 15 & Pulling shirt collar (away from neck) & 11 & $8 / 3$ \\
\hline 7 & Folding arms behind body & 126 & $82 / 44$ & 16 & $\begin{array}{l}\text { Playing with jewelry, and } \\
\text { manipulating other objects }\end{array}$ & 21 & $11 / 10$ \\
\hline 8 & Rubbing hands and crossing finger & 446 & $280 / 166$ & $\begin{array}{l}\text { Non- } \\
\text { MGs }\end{array}$ & Illustrative hand gestures & 1123 & $622 / 501$ \\
\hline 9 & Arms akimbo & 53 & $38 / 15$ & & Total number & 3692 & $2236 / 1456$ \\
\hline
\end{tabular}

TABLE II

STATISTICAL DISTRIBUTION OF MICRO-GESTURES AND NON-MICRO-GESTURES MANUALLY LABELED IN SMG. MG IDS CORRESPOND TO THE INDEXES IN FIGURE 2.
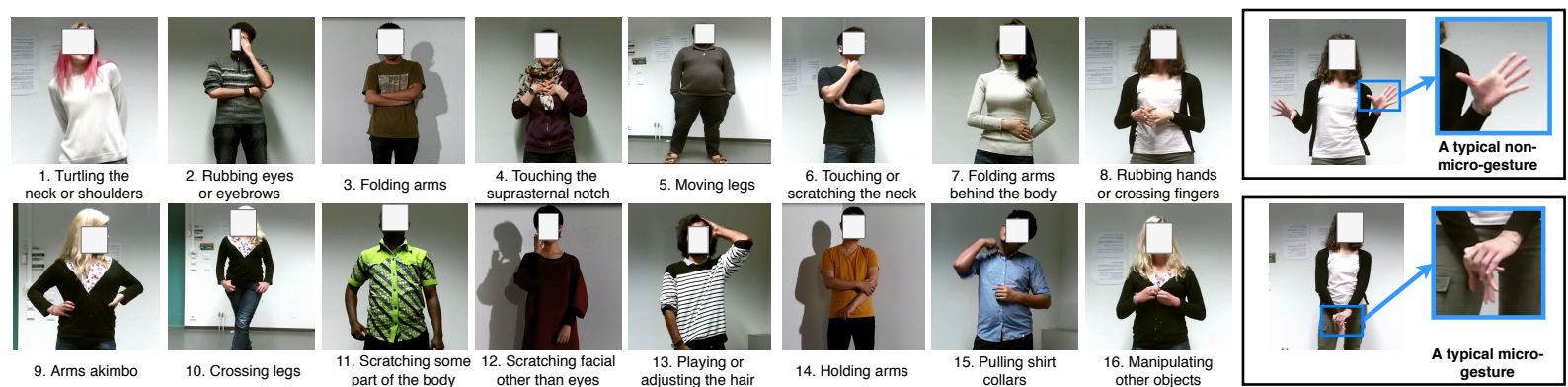

(a) A list of the micro-gesture in the SMG dataset

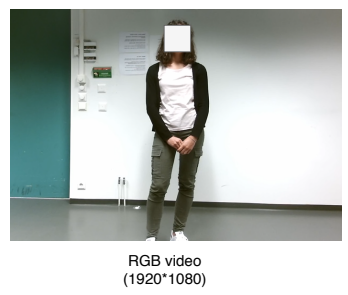

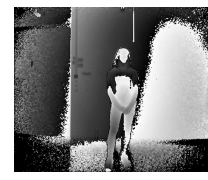

Depth Video
$\left(512^{*} 424\right)$

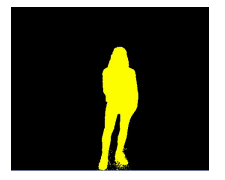

Silhouette
$\left(512^{*} 424\right)$

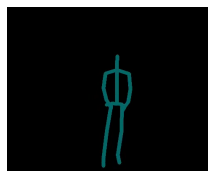

Skeleton
$(25$ coordinates)

(b) Sample frames of the four different modalities in the SMG dataset

Fig. 2. A list of all the MGs labeled in our SMG dataset. IDs and examples of corresponding MGs and non-micro-gestures are given. For the privacy concern, we mask the faces of the participants here.

used for recognizing emotional stress states via gestures. The emotion state recognition is a binary classification task to discriminate two emotional stress states (the RES vs. the SES).

\section{A. Relationship between MGs and emotional stress states}

First, an analysis was conducted to validate possible relationships between the MGs and the emotional stress states. The chi-square $\left(\chi^{2}\right)$ is computed as the measurement of the correlation, and the null hypothesis is given as MGs being distributed evenly in these emotional stress states. The result rejected the null hypothesis at $\left(\chi^{2}=164.79 ; p<0.001\right)$ level, and a significant correlation between emotional stress states and MGs is found. The analysis showed that the appearance frequency of body gestures of the participants and the emotional states involved were highly correlated.
Consequently, the result suggests that gestures could be used as cues to infer the emotion status of the two stress states.

\section{B. $M G$ recognition with deep learning}

For the recognition of MGs, we used sequences of 3D skeleton data as the inputs. We follow the work of [4] to adopt a Deep Belief Network (DBN) with a hidden Markov model (HMM) to detect and recognize the MGs in given sequences. We chose DBN for processing the skeleton data, as it has stacked restricted Boltzmann machines (RBMs) which can self-organize the joint coordinates. Firstly we modeled the temporal procedure of gestures with HMM by separating a gesture sequence into $T$ temporal segments. Then each segment was linked to one HMM state as shown in Figure 3.

For the visible layer in HMM, we denote the skeleton features as the observed variables $x_{t}$ which determines an 


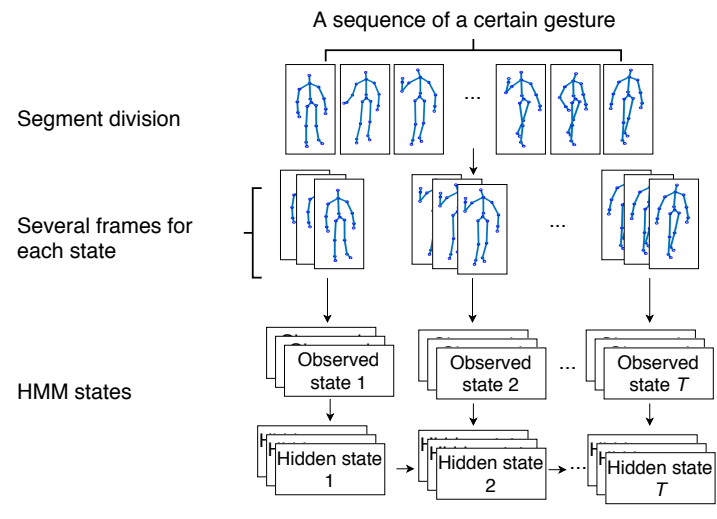

Fig. 3. The HMM model for processing a gesture. We averagely divide a given gesture into $T$ temporal segments as the HMM states.

observed state $X_{t}$. One observed state $X_{t}$ for $t=1, \cdots, T$ will be mapped to a corresponding hidden state as $H_{t}$ in the hidden layer of HMM. The hidden states are denoted as $h_{t}$; the transition among the hidden states was obtained training data. Based on the classical HMM framework, the full probability of HMM for training phase is specified as:

$$
\begin{array}{r}
p\left(x_{1}, x_{2}, \ldots, x_{T}, h_{1}, h_{2}, \ldots, h_{T}\right)= \\
p\left(h_{1}\right) p\left(x_{1} \mid h_{1}\right) \prod_{t=2}^{T} p\left(x_{t} \mid h_{t}\right) p\left(h_{t} \mid h_{t-1}\right)
\end{array}
$$

where $p\left(h_{1}\right)$ is the prior probability of each hidden state, $p\left(x_{t} \mid h_{t}\right)$ is the observation probability, namely, the emission matrix and $p\left(h_{t} \mid h_{t-1}\right)$ is the transition matrix. By pretraining DBN, the observation probability $p\left(x_{t} \mid h_{t}\right)$ can be obtained as:

$$
p\left(x_{t} \mid h_{t}\right)=p\left(h_{t} \mid x_{t}\right) p\left(x_{t}\right) / p\left(h_{t}\right)
$$

where $p\left(h_{t} \mid x_{t}\right)$ is the posterior probability of the HMM state. It is given by the trained DBN model. $p\left(h_{t}\right)$ is the prior probability of each HMM state. For $p\left(x_{t}\right)$, it is the prior probability of observed states which affects a lot when the sorts of the samples are seriously imbalanced.

In this work we implemented the DBN to estimate HMM states. We utilized the advantages of GaussianRBMs (GRBMs) for unsupervisedly initialing weights for feature layers and fine-tine the fully connected layers. We set our five-layer DBN model architecture as $\left[N_{X}, N_{h}, N_{h 1}, N_{h 2}, N_{\text {output }}\right]$. Here $N_{X}$ is the dimension of input features of the observation layer, $N_{h}$ is the number of hidden units in GRBM structure, and the DBN inner hidden layers between $N_{h}$ and $N_{\text {output }}$ are $N_{h 1}, N_{h 2}$. $N_{\text {output }}$ is the total HMM state number. In practical implementation, we trained the DBN with a structure of $[1000,1000,500]$.

After that the DBN was trained to give each frame an estimation of the hidden states with a SoftMax probability $p\left(h_{t} \mid x_{1}, x_{2}, \cdots, x_{t}\right)$. Viterbi algorithm was then used to decode sequence $\hat{g}$.

As a comparison to the $\mathrm{MG}$ recognition performance of DBN-HMM, we also implemented two basic sliding window-based recognition methods with a fully-connected network (FCN) and a DBN as baselines. A 3-layer FCN was used here with a structure of $[700,400,350]$. Sigmoid activation function was used without dropout in the layer. The DBN structure is exactly the same as the proposed structure above. At each given frame $t$, the frame was assigned with the class label that has the maximum probability. Once the frame level predictions were obtained by FCN and DBN separately, we employ a sliding window to generate the final recognition result. For both of the compared methods, the sliding window is set as five frames. If the frames within the window share the same gesture labels, one gesture would be allocated.

\section{An improved HMM transition strategy for MGs: Multiple Ergodic States}

Accurate segmentation of gesture sections is critical for the task of MGs detection and recognition from long sequences. To do this, it is common to recognize the 'nongesture' interval. Wu and Shao [28] called this interval as 'ergodic state' which was successfully utilized into HMM transition procedure. Once ergodic states in the sequences are determined, all the gestures could be segmented. However, ergodic states must exist between two gestures. In our spontaneous dataset, MGs usually occur continuously with no interval. For instance, several 'rubbing hands' MGs may occur in a row within a large snippet of unrelated illustrative gestures, and it is tough, even for human beings, to perfectly segment the 'rubbing hands' from 'illustrative hand gestures'. To address this problem, we proposed a novel method called Multiple Ergodic States (MES) with HMM to recognize MGs. We improved the ergodic-state method by treating not only the 'non-gesture' interval but also the start and end states of every gesture as ergodic states. It means we allow the transitions possible among all the MGs with multiple ergodic states (MES). In this way, the problem of consecutive MGs was tackled and the segmentation of several continuous incomplete gestures became possible. MES-HMM was proved to be considerably effective in recognizing continuous gestures in unconstrained long sequences as shown in Table III.

\section{A sign-based framework for emotional state recognition via gestures}

In the previous studies, emotion measurement using gestures were mostly message-based. This means that they assumed of one-to-one mappings of emotions and gestures whereas this assumption might be problematic in real-world applications. To tackle this problem, we propose a novel signbased measurement for gestural emotion recognition (not only for hidden emotion state). Instead of directly mapping one gesture to one emotion, we use a probability fashion for inference. The details of the framework are introduced below.

Consider a finite temporal sequence $X_{1: T}$ with a time length of $T$, observed from human-beings, it is the objective evidence for inferring the ultimate emotional state. Given an 


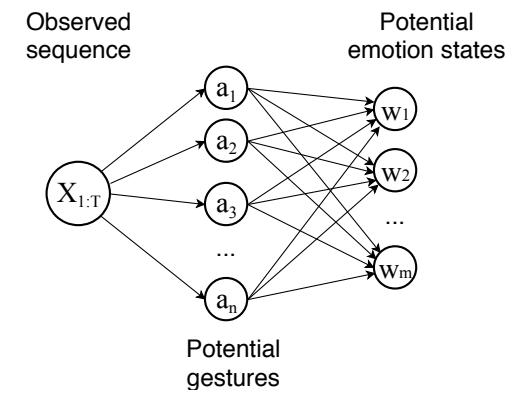

Fig. 4. The Bayesian Network used for the emotional state inference.

observed sequence $X_{1: T}, \underset{w_{i} \in C}{P}\left(w_{i} \mid X_{1: T}\right)$ is the target probability with different emotional states $w_{i}$, for $i=1, \cdots, N$. $N$ is the total number of emotional state classes. In our case, $\mathrm{N}=2$.

Then we have:

$$
\underset{w_{i} \in C}{P}\left(w_{i} \mid X_{1: T}\right)=\frac{P\left(w_{i}, X_{1: T}\right)}{P\left(X_{1: T}\right)}
$$

where $C$ is the set of all the stress states. Then, we introduced a set of intermediate variables $a_{j}$ for $j=1, \cdots, M$ which stands for the potential gestures. $M$ is the total number of gesture classes. The formulated expression becomes:

$$
\begin{array}{r}
P_{i \in C}\left(w_{i} \mid X_{1: T}\right)=\frac{\sum_{j=1}^{M} P\left(w_{i}, a_{j}, X_{1: T}\right)}{P\left(X_{1: T}\right)} \\
=\frac{\sum_{j=1}^{M} P\left(w_{i} \mid a_{j}, X_{1: T}\right) P\left(a_{j}, X_{1: T}\right)}{P\left(X_{1: T}\right)}
\end{array}
$$

Note that for $P\left(w_{i} \mid a_{j}, X_{1: T}\right), w_{i}$ and $X_{1: T}$ are conditionally independent when $a_{j}$ is determined, then formula (4) can be derived into:

$$
\begin{aligned}
\underset{w_{i} \in C}{P}\left(w_{i} \mid X_{1: T}\right)= & \frac{\sum_{j=1}^{M} P\left(w_{i} \mid a_{j}\right) P\left(a_{j}, X_{1: T}\right)}{P\left(X_{1: T}\right)} \\
& =\sum_{j=1}^{M} P\left(w_{i} \mid a_{j}\right) P\left(a_{j} \mid X_{1: T}\right)
\end{aligned}
$$

The distribution probability $P\left(w_{i} \mid a_{j}\right)$ stands for the confidence of the emotional stress state being $w_{i}$ with a given $a_{j}$. The conditional probability $P\left(a_{j} \mid X_{1: T}\right)$ stands for the probability of gesture $a_{j}$ in a given $X_{1: T}$. Now, with formula (5) we successfully set a mathematical mapping model from objective sequences to inner stress states via gestures. In this way, the probability relation of observations and emotional states was set with a Bayesian Network as shown in Figure 4. As shown in formula (5), we adopted a probability fashion to infer the human inner status (here is the stress state) with MGs.

$$
w_{i} \underset{\operatorname{argmax} w_{i}}{=} \sum \underset{w_{i} \in C}{P}\left(w_{i} \mid a_{j}\right) P\left(a_{j} \mid X_{1: T}\right)
$$

Eventually, based on the Bayesian rule we can further build a discriminative model for the emotional stress state inference as (6). Instead of crudely mapping from gestures to emotions. It makes the implementation of human hidden status recognition in the real world more smoothly.

Besides the framework of the DBN-HMM with Bayesian network (MG-Bayesian Network) we proposed above, we also trained a distinct DBN forthrightly to learn the emotional states as a reference (Forthright-DBN). We introduced this baseline to verify the capability of neural networks to learn emotional stress states directly from gestures (compared to the Bayesian network). So we used the same DBN structure (as the proposed framework) for the classification of the emotional stress states (instead of MGs). The outputs of the DBN are not MGs, but the stress states directly. Firstly, a 2layer FCN is used to achieve the gesture detection which is trained by all the labeled gestures. It has 2000 units at each layer with 'sigmoid' activation function. Then, each detected gesture in the given sequence is fed into the DBN and gives a probability of the RES and the SES. At last, the average emotion state probability estimated from all the gestures is used to predict the stress state of the given sequence.

\section{E. Experiment setting}

Performance of the proposed framework was evaluated using leave-subjects-out cross-validation with four folds. In each fold, ten subjects were taken for testing and the rest 30 subjects for training. RGB modality was not used as it might bring unnecessary texture patterns like facial information into neural networks and makes the analysis contested. We focus on the skeleton modality in order to specifically explore the relationship between gestures and stress states. Sequential Forward Floating Selection (SFFS) strategy were used for selecting MGs with the most contributions.

For skeleton joint feature extraction, we followed the work of [29]. Eigenjoints are features that utilize 3D position difference characters of joints to generate spatio-temporal information with a large dimension. Before feature extraction, the coordinates of the skeleton streams were smoothed by a Gaussian filter with Covariance set as 1.0. Then the skeletons were centrally normalized based on 'SpineBase' joints. Concerning the time issue, for each frame, we extracted Eigenjoint features with a dimension of 801 from selected 18 joints: 'ElbowLeft', 'WristLeft', 'ShoulderLeft', 'HandLeft', 'ElbowRight', WristRight', ShoulderRight', 'HandRight', 'Head', 'SpineShoulder', 'SpineBase', 'HipLeft', 'KneeLeft', 'AnkleLeft', 'HipRight', 'KneeRight', 'AnkleRight' and 'SpineShoulder'.

The feed-forward DBN network was pre-trained with a batch size of 100 . In the fine-tuning phase, the learning rate was set as 0.01 and it was trained with 20 epochs. For the FCN structure, the learning rate was set as 0.01 and it was trained with 12 epochs. The training time of a single DBN is around 2 hours with over 196,000 frames in our SMG dataset. The training platform was Theano with a single GPU: NVidia GTX 1080Ti (RAM: 12 GB).

\section{F. Human evaluation test}

As mentioned in the introduction, MGs are usually subtle and often neglected by humans in interactions. Thus using 


\begin{tabular}{llll}
\hline \hline Method & Precision & Recall & F1 score \\
FCN-Sliding window & 0.184 & 0.322 & 0.234 \\
DBN-Sliding window & 0.361 & 0.413 & 0.385 \\
DBN-ES-HMM & 0.272 & 0.456 & 0.341 \\
DBN-MES-HMM(our method) & $\mathbf{0 . 3 9 9}$ & $\mathbf{0 . 8 9 2}$ & $\mathbf{0 . 5 5 1}$ \\
\hline
\end{tabular}

TABLE III

Micro-GESTURE RECOGNITION RESUlt ON THE SMG DATASET

only MGs as the clues for stress state recognition should be a challenging task for human beings. We enroll human evaluators to test their ability to recognize stress states by observing the videos containing MGs, and then we compare human performance with those of our proposed computer models. Sixteen students were enrolled as testers. Another three evaluators who were trained to recognize MGs are also recruited to conduct the task. Skeleton and RGB modalities of all the 142 test sequences were offered separately to these testers in the form of videos (skeleton videos are played first, and then RGB videos follow). The evaluation results are shown in Table IV. The testers were also interviewed after the evaluation test. The majority of the testers claimed that it was hard to only use gestures (the skeleton modality) to infer the test sequences' stress states and it was actually a random guess. For RGB, people tend to use multiple cues such as facial expressions, micro-expressions, and even overall impressions (if the person is confident) to determine the stress states.

\section{RESUlTS AND DISCUSSION}

At first, we evaluate the performance of our framework for MG recognition. F1 score measurement is used to examine the MG recognition performance as shown in formula (7):

$$
F 1-\text { score }=\frac{2 \text { Precision } * \text { Recall }}{\text { Precision }+ \text { Recall }}
$$

The Precision is the fraction of relevant MGs among all the gestures retrieved in a test sequence by machines, while Recall (or sensitivity) is the fraction of MGs that have been retrieved over the total amount of annotated MGs in that test sequence. Recognition results of the MGs with 'micro-gesture based' framework are shown in Table III. For both detection and recognition tasks, the DBN structure performs better than the FCN ( 0.151 in the F1 score) as shown in the first two lines in Table III. The HMM temporal modeling strategy of the DBN-ES-HMM framework contributes 0.043 improvements in 'Recall' but brings 0.089 decreases in 'Precision' when compared to the DBN-Sliding window framework. The reason might be that the HMM could allow the transition of the gestures in a sequence with soft probability. So it could recall more gestures while brought many noises. While the sliding window just selects the most confident results, which is a hard-coded behavior. The performance of our MES-HMM is much better than that of ES-HMM (with the improvement by $21.0 \%$ in the F1 score). The diverse transition of MES decreased the noises in the HMM transition and brought significant improvement.

\begin{tabular}{|c|c|c|c|c|c|}
\hline \multirow{2}{*}{ Tester } & \multirow{2}{*}{ Modality } & \multirow{2}{*}{ Prediction } & \multicolumn{2}{|c|}{ Emotion State } & \multirow{2}{*}{ Accuracy } \\
\hline & & & RES & SES & \\
\hline \multirow{4}{*}{$\begin{array}{l}\text { Common } \\
\text { People }\end{array}$} & \multirow{2}{*}{ Skeleton } & RES & 36.1 & 34.9 & \multirow{2}{*}{0.480} \\
\hline & & SES & 38.9 & 32.1 & \\
\hline & \multirow[b]{2}{*}{ RGB } & RES & 39.2 & 31.8 & \multirow{2}{*}{0.538} \\
\hline & & SES & 33.8 & 37.2 & \\
\hline \multirow{4}{*}{$\begin{array}{l}\text { Trained } \\
\text { Evaluators }\end{array}$} & \multirow{2}{*}{ Skeleton } & RES & 40.5 & 30.5 & \multirow{2}{*}{0.655} \\
\hline & & SES & 18.5 & 52.5 & \\
\hline & \multirow{2}{*}{ RGB } & RES & 48 & 23 & \multirow{2}{*}{0.745} \\
\hline & & SES & 13.2 & 57.8 & \\
\hline
\end{tabular}

TABLE IV

Human EVALUATION RESUlt. RES = RELAXED EMOtion STATE, SES $=$ STRESSED EMOTION STATE

\begin{tabular}{|c|c|c|c|c|}
\hline \multirow{2}{*}{ Framework } & \multirow{2}{*}{ Prediction } & \multicolumn{2}{|c|}{ Emotion State } & \multirow{2}{*}{ Accuracy } \\
\hline & & RES & SES & \\
\hline \multirow{2}{*}{ MG-Bayesian Network } & RES & 36 & 34 & \multirow{2}{*}{0.549} \\
\hline & SES & 30 & 42 & \\
\hline \multirow{2}{*}{ MG-Bayesian Network (SFFS) } & RES & 33 & 38 & \multirow{2}{*}{0.606} \\
\hline & SES & 18 & 53 & \\
\hline \multirow{2}{*}{ Forthright-DBN } & RES & 50 & 6 & \multirow{2}{*}{0.521} \\
\hline & SES & 62 & 24 & \\
\hline \multirow{2}{*}{ GT-Bayesian Network (as reference) } & RES & 37 & 13 & \multirow{2}{*}{0.632} \\
\hline & SES & 34 & 58 & \\
\hline
\end{tabular}

TABLE V

EMOTION RECOGNITION RESULTS OF PROPOSED METHODS WITH ONLY GESTURES. RES = RELAXED EMOTION STATE, SES = STRESSED EMotion State, GT = Ground Truth Micro-GESTURE

Eventually, emotional stress state recognition performance of the proposed frameworks is shown in Table V. It is evident that 'MG-Bayesian Network (SFFS)' framework performs much better (0.606) than both human test $(0.480)$ and 'Forthright-DBN' framework (0.521). But when it comes to the trained evaluators $(0.655)$, human beings performed still better than our framework. It is worthy to mention that, during the training of 'Forthright DBN', the network loss was extremely hard to converge, which means, so far, neural networks are still not competent for the task of recognizing the relatively high-level targets, such as emotional stress states. It also worth mentioning that, gestures of 'Turtling neck or shoulders', 'Crossing legs', 'Scratching facial other than eyes' and 'Pulling shirt collars' are selected automatically for their offering the contributions to the best overall recognition performance. It is very interesting that the framework has potential in helping people to understand and implement MGs better.

We also used manually labeled MGs with Bayesian Networks to infer the stress states as a reference (0.632) shown in the last line of Table V. It could be regarded as the ultimate correlation between MGs with stress states that can be observed by human beings. Although the result of our framework superiors to common observers, it is far from reliable performance. It might be caused by the insufficient contrast between the two emotional states due to the attitude of the participants. The differences of gesture expressions and cultures between the 40 participants can also largely affect the statistics needed by the Bayesian network. A more 
reliable model to infer emotional states from gestures is desirable in the future. In other words, although MGs and human inner status are strongly related, using only MGs is not enough to get a very high recognition result of hidden emotional states.

\section{CONCLUSIONS AND FUTURE WORKS}

The work in this paper showed that body gestures are helpful clues for human hidden emotion recognition. For the task of exploring the potential relation between the MGs and human hidden stress states with machine vision methods, we made several meaningful contributions: 1) We studied hidden-emotion related gestures, so-called MGs, and the correlation between the human stress states and the gestures was investigated and proved by us. To the best of our knowledge, this is the first time that MGs are investigated with computer vision methods. 2) We proposed a novel Spontaneous MicroGesture dataset, which includes 3,692 multi-stream gesture clips elicited from 40 participants under two emotional stress states. 3) A framework with sign-based measurement for mappings from real-world observations to human hidden status was proposed, which makes the implementation of human inner status recognition in the real world more smoothly. 4) Two different deep-learning frameworks ('MG-Bayesian Network' framework and 'Forthright DBN' framework) were offered to tackle the task of the human emotional state recognition with gesture observations. Baseline performances are also provided with enhanced methods (MES-HMM). We got a very promising result using MGs for emotional stress state recognition which exceeds human test results (common people) by $12.6 \%$.

In future, more non-verbal communicative cues such as facial expressions can be combined with MGs using the RGB modality in the SMG dataset for improving the emotional recognition performance. Efforts will be made to improve the 'Forthright-DBN' framework into a more efficient endto-end framework.

\section{ACKNOWLEDGMENT}

This work was supported by the Academy of Finland, MiGA project (Grant No.316765), Tekes Fidipro Program (Grant No.1849/31/2015) and Business Finland project (Grant No. 3116/31/2017). Haoyu Chen is supported by China Scholarship Council. As well, the authors wish to acknowledge CSC IT Center for Science, Finland, for computational resources.

\section{REFERENCES}

[1] H. Aviezer, Y. Trope, and A. Todorov. Body cues, not facial expressions, discriminate between intense positive and negative emotions. Science, 338(6111):1225-1229, 2012.

[2] R. Axtell. Gestures: The Do's and Taboos of Body Language Around the World. Wiley, 1997.

[3] J. Burgoon, D. Buller, and W. W.G. Nonverbal communication: The unspoken dialogue. Columbus, OH: Greyden Press, 1994.

[4] H. Chen, X. Liu, and G. Zhao. Temporal hierarchical dictionary with HMM for fast gesture recognition. In 24th International Conference on Pattern Recognition, ICPR 2018, Beijing, China, August 20-24, 2018, pages 3378-3383, 2018
[5] J. Cohn and P. Ekman. Measuring facial action by manual coding, facial emg, and automatic facial image analysis. In . K. S. J.A. Harrigan, R. Rosenthal, editor, Handbook of nonverbal behavior research methods in the affective sciences. October 2003.

[6] P. Collett. The book of tells: From the bedroom to the boardroom how to read other people. Ontario: HarperCollins Ltd, 2003.

[7] N. Dael, M. Mortillaro, and K. R. Scherer. The body action and posture coding system (bap): Development and reliability. Journal of Nonverbal Behavior, 36(2):97-121, 2012.

[8] G. de Becker. The gift of fear. New York: Dell Publishing, 1997.

[9] J. Dimitrius and M. Mazzarella. Put your best foot forward: Make a great impression by taking control of how others see you. New York: Fireside, 2002.

[10] P. Ekman. Basic emotions. Handbook of cognition and emotion, pages 45-60, 1999.

[11] R. Ekman. What the face reveals: Basic and applied studies of spontaneous expression using the Facial Action Coding System (FACS). Oxford University Press, USA, 1997.

[12] B. Gelder, J. Stock, H. Meeren, C. Sinke, M. Kret, and M. Tamietto. Standing up for the body. recent progress in uncovering the networks involved in the perception of bodies and bodily expressions. Neuroscience and Biobehavioral Reviews, 34(4):513 - 527, 2010.

[13] C. Ginevra, K. Loic, and C. George. Emotion Recognition through Multiple Modalities: Face, Body Gesture, Speech, pages 92-103. Springer Berlin Heidelberg, Berlin, Heidelberg, 2008.

[14] Y. Gu, X. Mai, and Y. Luo. Do bodily expressions compete with facial expressions? time course of integration of emotional signals from the face and the body. PLOS ONE, 8(7):1-9, 072013.

[15] H. Gunes and M. Piccardi. A bimodal face and body gesture database for automatic analysis of human nonverbal affective behavior. In 18th International Conference on Pattern Recognition, volume 1, pages 1148-1153, 2006.

[16] H. Gunes, M. Piccardi, and M. Pantic. From the Lab to the real world: affect recognition using multiple cues and modalities, pages 185-218. Number 2008/16200. InTech Education and Publishing, 2008.

[17] M. Knapp and J. Hall. Nonverbal Communication in Human Interaction. Harcourt Brace College Publishers, New York, 1972.

[18] M. Krakovsky. Artificial (emotional) intelligence. Commun. ACM, 61(4):18-19, Mar. 2018.

[19] E. Kuhnke. Body Language For Dummies. Wiley, 2009.

[20] X. Li, T. Pfister, X. Huang, G. Zhao, and M. Pietikainen. A spontaneous micro-expression database: Inducement, collection and baseline. In 2013 10th IEEE International Conference and Workshops on Automatic Face and Gesture Recognition (FG), pages 1-6, April 2013.

[21] A. Mohanty and R. Sahay. Rasabodha: Understanding indian classical dance by recognizing emotions using deep learning. Pattern Recognition, 79:97 - 113, 2018.

[22] J. Navarro and M. Karlins. What every BODY is saying : an ex-FBI agent's guide to speed reading people. Collins, New York, 2008.

[23] E. Paul and O. Maureen. Who can catch a liar? 46:913-20, 101991.

[24] A. Pentland. Honest Signals: How They Shape Our World. MIT Press, Cambridge, MA, 2008

[25] M. Radeta and M. Maiocchi. Towards automatic and unobtrusive recognition of primary-process emotions in body postures. In 2013 Humaine Association Conference on Affective Computing and Intelligent Interaction, pages 695-700, Sept 2013.

[26] G. Serge. International glossary of gestalt psychotherapy. In FORGE, 1995.

[27] A. Vinciarelli, M. Pantic, and H. Bourlard. Social signal processing: Survey of an emerging domain. Image and Vision Computing, 27(12): 1743 - 1759, 2009.

[28] D. Wu and L. Shao. Leveraging hierarchical parametric networks for skeletal joints based action segmentation and recognition. In 2014 IEEE Conference on Computer Vision and Pattern Recognition, pages 724-731, June 2014.

[29] X. Yang and Y. L. Tian. Eigenjoints-based action recognition using naive-bayes-nearest-neighbor. In 2012 IEEE Computer Society Conference on Computer Vision and Pattern Recognition Workshops, pages 14-19, 2012.

[30] Z. Zeng, M. Pantic, G. I. Roisman, and T. S. Huang. A survey of affect recognition methods: Audio, visual, and spontaneous expressions. IEEE Transactions on Pattern Analysis and Machine Intelligence, 31(1):39-58, Jan 2009.

[31] C. Zhang and Y. Tian. Subject adaptive affection recognition via sparse reconstruction. In 2014 IEEE Conference on Computer Vision and Pattern Recognition Workshops, pages 357-364, June 2014. 